\title{
Letter
}

\section{Notes on the Amplification of Magnetic Field Variations on the Lunar Surface}

\author{
(Received January 4, 1971)
}

Data obtained by the Lunar Surface Magnetometer (LSM) which was set by the Apollo 12 Mission have clearly shown that amplitudes of magnetic field variations are appreciably amplified on the lunar surface in comparison with the ambient field variations. Figure 1 shows an example of the comparison of simultaneous data of $N-S$ component of magnetic field variation recorded by LSM and by a magnetometer on Explorer 35 (Dyal et al., 1970). In case of this example, both magnetometers were located in the sunlit side with respect to the moon. As seen in the figure, both magnetograms are in good agreement with each other in regard to their phases, but the amplitudes of variation recorded by LSM are much larger than those recorded by the Explorer 35 magnetometer. Here, the quasi-periodic variation during about 7 minutes from 33 to 40 minutes in time in the figure will be specifically dealt with.

The average period of the quasi-periodic variation was 102 seconds. The ratio of amplitude of each wave variation for a half wave length in LSM magnetogram to that
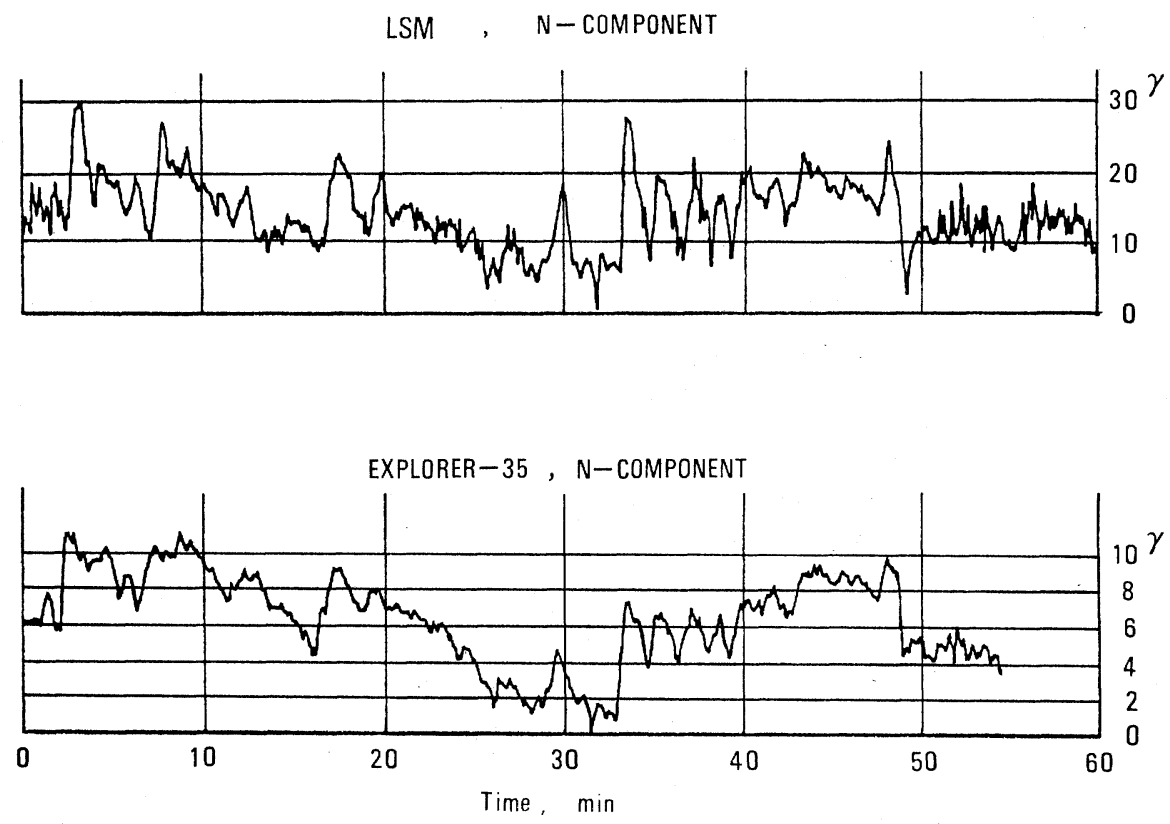

Fig. 1 Example of LSM magnetogram in comparison with simultaneous magnetogram obtained on Explorer 35 (Rearranged from data published by Dyal, Parkin and Sonett). 
in Explorer 35 magnetogram ranges from 3.3 to 5.5, the average value being about 4.7 . The wave forms of the field variation observed by the two magnetometers are exactly the same within the accuracy of time-resolution in both magnetometers. Then, this fact may imply that the magnetic field variations are amplified particularly on the moon's surface.

A possibility of enhancement of magnetic field variations on the lunar surface has already been suggested by several workers (e.g., Blanck and Sill, 1969; Schwartz and Schubert, 1969; Schubert and Schwartz, 1969). More generally the electromagentic interaction between the moon and the solar wind has been discussed by several other theoretical investigators also (e.g., Sonett and Colburn, 1967; Johnson and Midgley, 1968; Hollweg, 1968; Schwartz et al., 1969). It does not seem however that the practical aspect of the electromagnetic interaction between the móon and the solar plasma wind has already been clearly understood.

The toroidal magnetic field $(\vec{B} t)$ generated by the moon-solar wind interaction is caused by $\vec{E}_{t}=\vec{v} \times \vec{B}_{0}$ electromotive force, where $\vec{v}$ denotes the relative velocity between the moon and the solar wind and $\vec{B}$ o represents the average magnetic force in the solar wind. Because the electrical conductivity of the moon's interior is finite and that of the solar wind is very large, a closed circuit of electric current could be formed between the moon and the solar wind plasma. In this case, the intensity of electric current $(I)$ should be essentially subjected to the total electrical resistance $(R)$ of the moon in accordance with Ohm's law, $I=E_{t} \mid R$. Here $R$ is given by $\int r(s) d s$, where $r(s)$ and $s$ denote respectively the resistivity at $s$ and the distance along a closed circuit of electrical current flow.

The electrical resistivity $r(s)$ within the moon's interior is sensitively dependent on temperature at $s$. The electrical conductivity $\sigma=1 / r$ of Apollo 11 and 12 lunar materials dependent on temperature has been measured by Nagata et al. (1970) and Schwerer et al. (1971). A summary of their results is illustrated in Fig. 2. The essential difference of the electrical conductivity of the lunar materials from that of terrestrial rocks is that the former is much larger than the latter by two or three in the order of magnitude throughout the whole range of temperature, probably because of much more abundance of free iron ions $\left(\mathrm{Fe}^{2+}\right)$ in the former.

However, the total resistance along the moon's diameter is estimated to be as large as $3.0 \times 10^{15} \Omega / \mathrm{cm}^{-2}$, and the average conductivity of the moon's interior is about $4.3 \times$ $10^{-8}(\Omega \mathrm{cm})^{-1}$, even if the largest considerable thermal gradient (i.e., a thermal model of chondritic moon of 4.5 billion years in age; England et al., 1968) is assumed for the moon's interior, where the temperature at the lunar center is estimated to be $2,100^{\circ} \mathrm{K}$. This is because of the low electrical conductivity of the outermost part of the moon's interior. It may thus be concluded that the electromagnetic shock wake cannot be generated by the moon, even though the electrical conductivity of the moon's interior is extremely high.

The other magnetic field generated by the electrically conductive moon is the timevarying poloidal field, which is essentially due to the electric currents electromagnetically 


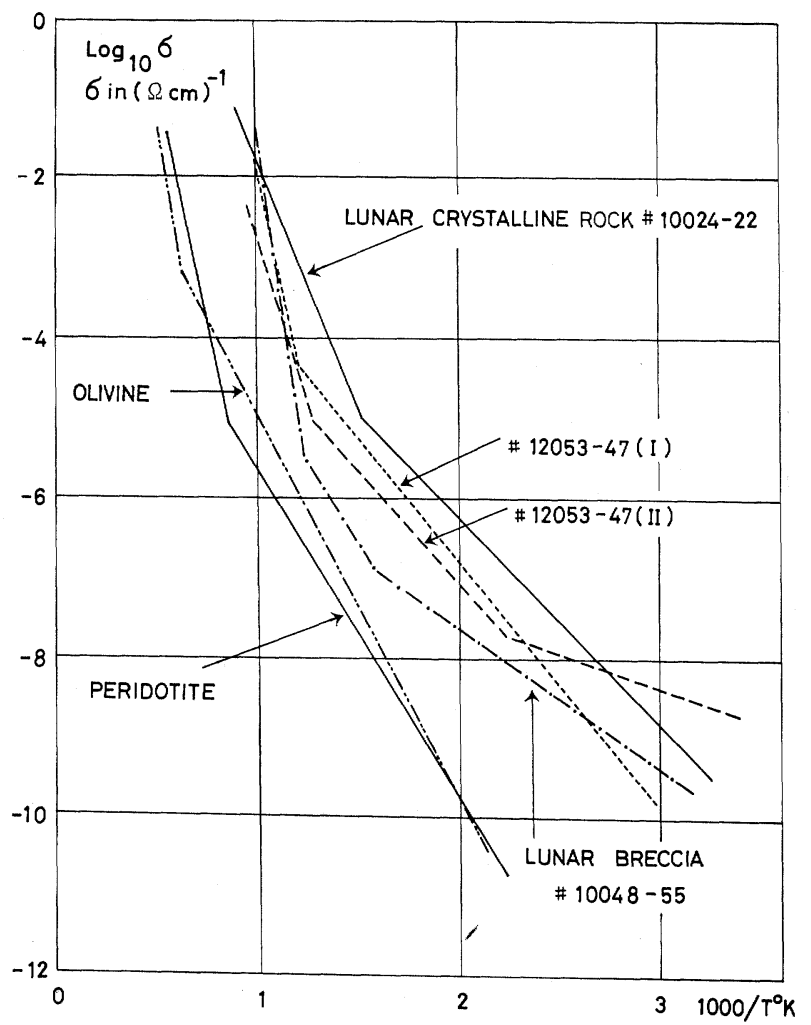

Fig. 2 Electrical conductivity of Apollo 11 and 12 lunar materials as dependent on temperature in comparison with that of typical terrestrial rocks (after Schwerer, Nagata and Fisher) Lunar samples \#10024-22: Apollo 11 crystalline rock \# 12053-47 (I): Apollo 12 crystalline rock \#12053-47 (II): Another specimen cut from the same sample as above

\#10048-55: Apollo 11 microbreccia

induced within the moon. The electromagnetic induction is simply expressed in term of the vector potential $\vec{A}$ in a form of $\nabla^{2} \vec{A}=4 \pi \mu \sigma \frac{\partial}{\partial t} \vec{A}$, where $\mu$ denotes the magnetic permeability. It must be taken into account however that the moon is directly surrounded by the electrically high conductive plasma of the solar wind, and consequently the timevarying poloidal magnetic field caused by the electric current induced within the conductive moon's interior must be suppressed by the kinetic pressure of the surrounding solar wind plasma. This situation has already been clearly described by Schubert and Schwartz (1969) in their theoretical treatise. Let us re-express their mathematical expressions in a more intuitive physical picture in this note.

Insofar as dealing with the time-varying magnetic field of $10^{2} \mathrm{sec}$ in period, such as shown in Fig. 1, the electrical conductivity of the solar wind plasma $\left(\sigma_{s}\right)$ may be approximated to be infinitely large, because $\sigma_{s} \gtrsim 10^{2}(\Omega \mathrm{cm})^{-1}$. 
Experimental results obtained by Nagata et al. (1970) have indicated that the average electrical conductivity $\left(\bar{\sigma}_{o}\right)$ of the moon's uppermost shell of $200 \mathrm{~km}$ in thickness is given by $\bar{\sigma}_{o}=1.4 \times 10^{-8}(\Omega \mathrm{cm})^{-1}$, whereas the electrical conductivity of the moon's deep interior $\left(\sigma_{i}\right)$ in depth deeper than $200 \mathrm{~km}$ is larger than $5 \times 10^{-2}(\Omega \mathrm{cm})^{-1}$. Then $\bar{\sigma}_{o}$ can be regarded negligiblly small compared with $\sigma_{i}$ as well as $\sigma_{s}$. Then, as far as the periodic change of magnetic field of $10^{2} \mathrm{sec}$ in period is concerned, we may be able to assume a simple electrical model picture of the moon surrounded by the high conductive solar wind as illustrated in Fig. 3, where $\sigma_{s} \simeq \infty, \sigma_{0} \simeq 0$ and $\sigma_{i} \simeq \infty$. This model would indicate that the induced time-varying magnetic field $\left(\vec{B}_{i}\right)$ must be confined to a thin non-conductive shell of the moon, which is sandwiched between the high conductive solar wind flow and the high conductive interior of the moon on the sunlit side.

Based on the simple model, the mechanism of amplification of time-varying magnetic field on the moon's sunlit surface may be formulated in the following expressions.

Assume that the magnetic field $(\vec{B})$ in the solar wind is expressed by

$$
\vec{B}=\vec{B}_{0} e^{j \omega t} \text {. }
$$

$$
B_{0} e^{j \omega t}
$$

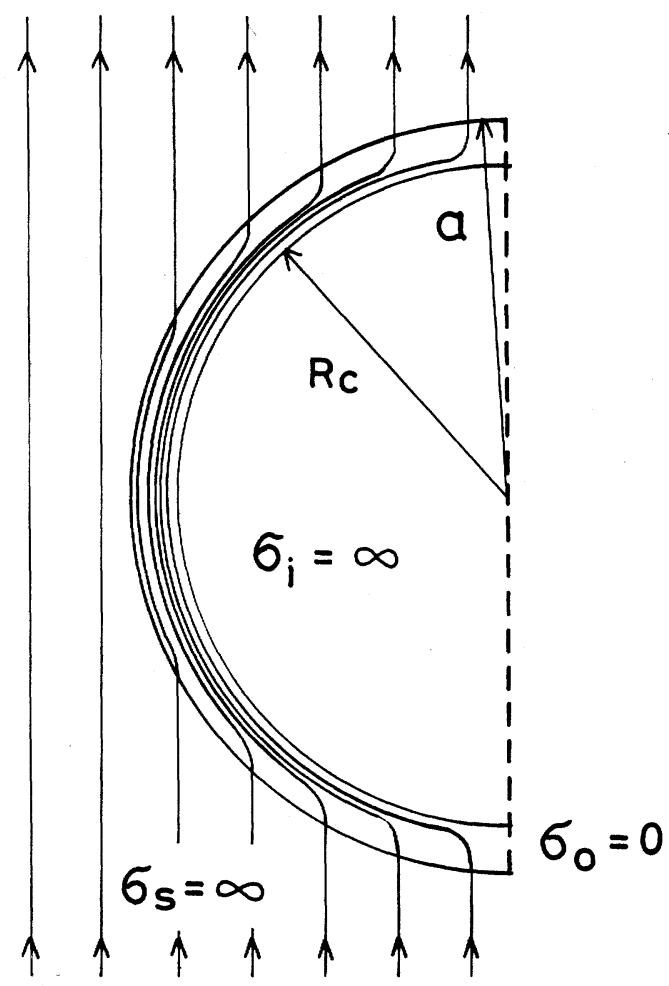

Fig. 3 Model of the sunlit side of the moon for a periodic change of a spatially uniform and time-varying magnetic field $B_{0} \exp (j \omega t)$ in the solar wind. 
The time-varying magnetic field penetrating into the non-conductive outer shell of the moon is to be reflected by the outer boundary of the conductive core as well as the moon's surface surrounded by the high conductive solar wind, provided that the magnetic pressure is smaller than the solar wind pressure at the lunar surface. Then, the radial $\left(B_{s r}\right)$ and tangential $\left(B_{s \theta}\right)$ components of the magnetic force on the sunlit lunar surface is expressed by

$$
\begin{aligned}
& B_{s r}=-B_{0} e^{j \omega t} \cos \theta \\
& B_{s \theta}=B_{0} e^{j \omega t}\left(1+\frac{3}{2} \frac{\Delta^{3}}{1-\Delta^{3}}\right) \sin \theta, \quad \text { on } \quad r=a,
\end{aligned}
$$

where

$$
\Delta \equiv r_{c} / a \quad \text { and } \quad \vec{B} s=\left(B_{s r}, B_{s \theta}\right)
$$

LSM data have shown that $(B s / B o) \simeq 5$ at $\theta \simeq \frac{\pi}{2}$. Then equation (2) leads to $\Delta \simeq 10 /$ 11. This result indicates that the thickness of the non-conductive outer shell is about $160 \mathrm{~km}$.

In the neighbourhood of the subsolar point on the lunar surface, the solar wind pressure is approximately equivalent to the magnetic pressure of $60 \gamma$ in the force intensity. Since $B 0 \leq 6 r$ and $B s\left(\theta=\frac{\pi}{2}\right) \leq 30 \gamma$ in case of Fig. 1, the magnetic pressure of the amplified time-varying magnetic field in the lunar outer shell is smaller than the solar wind pressure, whence the amplified magnetic field is confined to the non-conductive outer shell of the moon. In the dark side of the moon, however, the magnetic field may be expanded backwards, because the inward pressure of the surrounding plasma should be much less in the dark side, being approximately equivalent to the magnetic pressure of several gammas. Thus, the amplification ratio may be only two in the largest possible case on the dark lunar surface, where the ambient magnetic field $B o$ is superposed by a dipole magnetic field $B^{\prime}$ which is due to the electric current induced within the conductive core.

\section{References}

Blanck, J.L. and W.R. Sill, Response of the Moon to the Time-Varying Interplanetary Magnetic Field J. Geophys. Res., 74, 736-743, 1969.

Dyal, P., W. Parkin and C.P. Sonett, Lunar Surface Magnetometer Experiment. Apollo 12 Preliminary Science Report (NASA SP-235), 55-73, 1970.

England, A.W., G. Simmons and D.W. Strangway, Electrical Conductivity of the Moon. J. Geophys. Res., 73, 3219-3226, 1968.

Hollweg, J.V., Interaction of the Solar Wind with the Moon and Formation of a Lunar Limb Shock Wave. J. Geophys. Res., 73, 7269-7276, 1968.

Johnson, F.S. and J.E. Midgley, Notes on the Lunar Magnetosphere. J. Geophys. Res., 73, 1523-1532, 1968. Nagata, T.,T. Rikitake and M. Kono, Electrical Conductivity and the Age of the Moon. Space Research Vol. XI, 85-88, 1971.

Schubert, G. and K. Schwartz, A theory for the Interpretation of Lunar Surface Magnetometer Data, Moon, 1, 106-117, 1969. 
Schwartz, K. and G. Schubert, Time Dependent Lunar Electric and Magnetic Fields Induced by a Spatially Varying Interplanetary Magnetic Field. J. Geophys. Res., 74, 4777-4780, 1969.

Schwartz, K., C.P. Sonett and D.S. Colburn, Unipolar Induction in the Moon and A Lunar Limb Shock Mechanism. Moon, 1, 7-30, 1969.

Schwerer, F.C., T. Nagata and R.M. Fisher, Electrical Conductivity of Lunar Surface Rocks and Chondritic Meteorites. Moon, 3, (1971) (in press).

Sonett, C.P. and D.S. Colburn, Establishment of a Lunar Unipolar Generator and Associated Shock and Wake by the Solar Wind. Nature, 216, 340-343, 1967.

Takesi NAgAtA

Geophysics Research Laboratory

University of Tokyo 